\title{
Mechanical Thrombectomy for ICA Toport Occlusion with Twig-Like MCA: A Case Report
}

Yu Sakai, Gakushi Yoshikawa, and Katsuya Sato

Objective: We report a case of hemorrhagic complication after mechanical thrombectomy (MT) for internal carotid artery (ICA) occlusion with twig-like middle cerebral artery (MCA).

Case Presentation: A 75-year-old man was admitted to our hospital with ICA occlusion. Recanalization was achieved by a direct aspiration first pass technique (ADAPT). The peripheral MCA was twig-like, but operators thought that a thrombus remained in the MCA first segment. The procedure was continued and suspended with perforation of the microguidewire.

Conclusion: When performing MT for large vessel occlusion (LVO) with twig-like MCA, it is difficult to proceed a device to the periphery and there is a risk of hemorrhage.

Keywords > twig-like middle cerebral artery, mechanical thrombectomy, hemorrhagic complication

\section{Introduction}

Twig-like middle cerebral artery (MCA) is a relatively rare entity. ${ }^{1)}$ Twig-like MCA causes hemorrhagic stroke and ischemic stroke, but to our knowledge, no case of major cerebral artery occlusion complicating twig-like MCA treated by thrombectomy has been reported. We report a patient with twig-like MCA in whom cardiogenic terminal occlusion of the internal carotid artery (ICA) developed on the ipsilateral side and was treated by percutaneous thrombectomy, which led to recanalization and accompanying hemorrhagic complication.

\section{Case Presentation}

The patient was a 75-year-old man with no medical history or oral medications. Modified Rankin Scale (mRS) before

Department of Neurosurgery, Showa General Hospital, Kodaira, Tokyo, Japan

Received: November 27, 2020; Accepted: June 11, 2021 Corresponding author: Yu Sakai. Department of Neurosurgery, Showa General Hospital, 8-1-1, Hanakoganei, Kodaira, Tokyo 187-8510, Japan

Email: sakaiyu1210@gmail.com

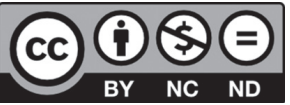

This work is licensed under a Creative Commons Attribution-NonCommercialNoDerivatives International License.

(C)2021 The Japanese Society for Neuroendovascular Therapy the onset was 0 . The patient was transported to our hospital because of left paralysis approximately 2 hours after onset. The neurological findings on arrival were complete paralysis of the left half of the body, rightward conjugate deviation, and left spatial neglect, and the National Institutes of Health Stroke Scale (NIHSS) ${ }^{2}$ was 16. Atrial fibrillation was detected on electrocardiography at the time of arrival. On MRI, the Alberta stroke program early CT score diffusion weighted imaging (DWI) (ASPECTS-DWI) ${ }^{3)}$ was 4 (Fig. 1A and 1B), and right ICA occlusion was noted on MRA (Fig. 1C). Vascular hyper-intensity4) was observed in the distal M1 region on FLAIR (Fig. 1D). As the change in the intensity on DWI was weak, thrombus retrieval was selected in consideration of reversibility. Tissue plasminogen activator was not administered in consideration of the risk of hemorrhagic complication.

On contrast imaging using an Optimo 9 Fr (Tokai Medical Products, Aichi, Japan), the right ICA was occluded in the region distant from the ophthalmic artery bifurcation, suggesting terminal occlusion of the ICA (Fig. 2A and 2B). A Penumbra 5MAX ACE68 (Penumbra, Alameda, CA, USA) was advanced to the proximal part of the lesion and placed where the suction stopped. At this time, no microguidewire or microcatheter crossed the lesion. When the balloon of the Optimo was inflated and the Penumbra was retrieved while manually applying suction, a red thrombus was present. On contrast imaging, effective recanalization of modified thrombolysis in cerebral infarction (mTICI) ${ }^{5}$ 


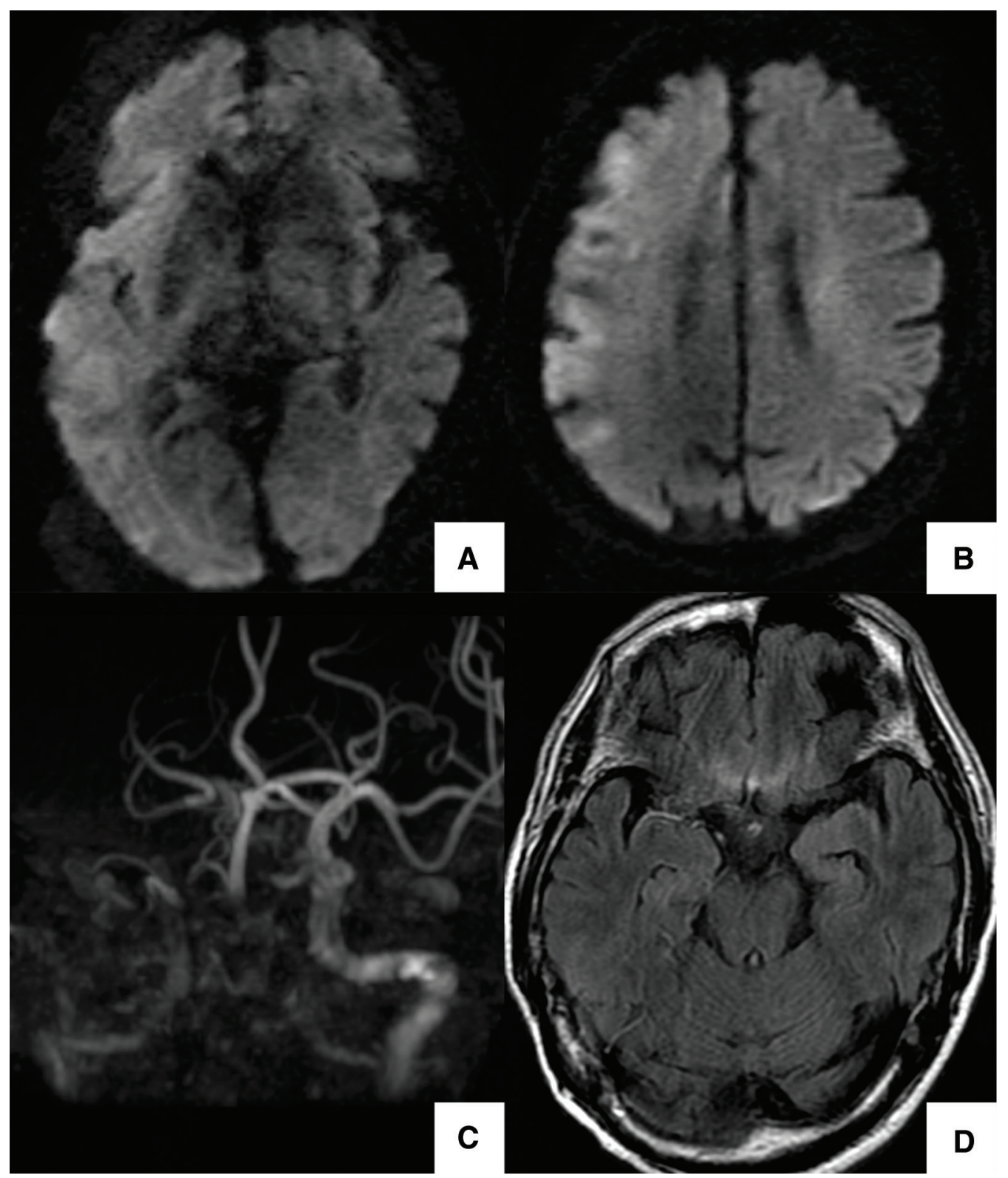

Fig. $1 \mathrm{MRI}$ at onset. The DWI-ASPECTS was 4 on DWI (A and B). On MRA, occlusion of the right internal carotid artery was noted (C). On FLAIR, the right MCA was visualized as a thin high-intensity signal (D). ASPECTS: Alberta stroke program early CT score; DWI: diffusion weighted imaging; MCA: middle cerebral artery

2b was achieved (Fig. 2C and 2D). Although recanalization was effective at this time point, the operators continued the procedure in consideration of remaining thrombus in the proximal region of the MCA first segment (M1). Lesion crossing with a microguidewire to the lesion considered present in the proximal M1 region was carried out using the Optimo, Penumbra 5MAX ACE68, Marksman (Medtronic, Minneapolis, MN, USA), and CHIKAI black soft tip 14 (Asahi Intecc, Aichi, Japan), which caused perforation (Fig. 3A) and a small volume of extravasation was observed (Fig. 3B). When the blood pressure was reduced by nicardipine, the anticoagulant was neutralized with $50 \mathrm{mg}$ of protamine, an image was acquired after waiting for several minutes, and extravasation disappeared. On cone-beam CT, a small volume of subarachnoid hemorrhage (SAH) was noted (Fig. $3 \mathbf{C}$ ). At this time point, the operators confirmed that thrombus was absent in M1 and the region was a twig-like MCA, completing the procedure (Fig. 3D-3F). The time from puncture to recanalization (P2R) was $28 \mathrm{~min}$. On head MRI on the second hospital day, the ASPECTS-DWI was 2 (Fig. 4A and 4B) and ICA occlusion had resolved on MRA (Fig. 4C). The NIHSS 


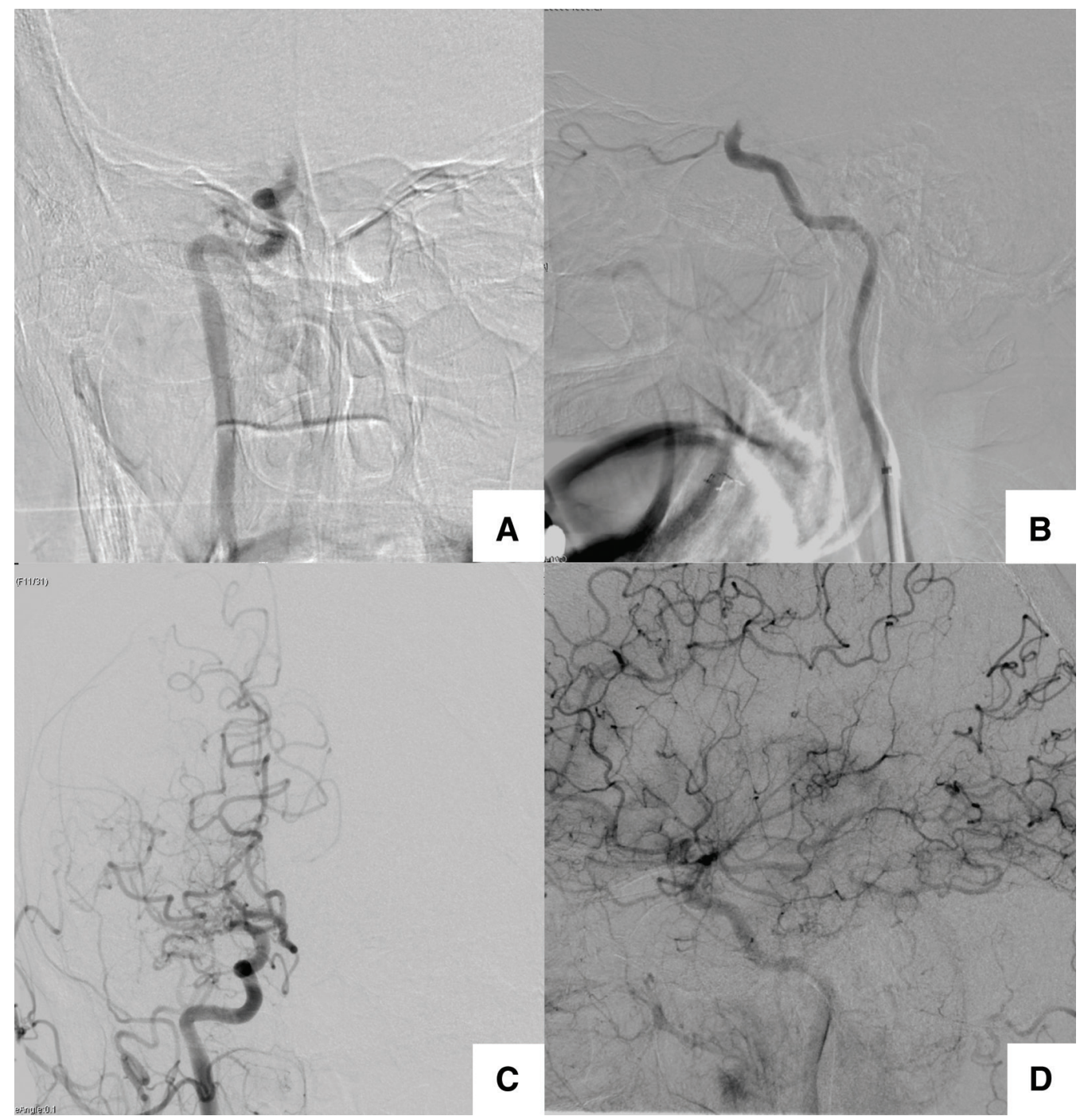

Fig. 2 Angiography at the time of thrombus retrieval. Frontal and lateral views of the blood vessel before thrombectomy (A and B). A thrombus was located distal to the bifurcation of the ophthalmic artery from the internal carotid artery. The frontal and lateral views on angiography after 1 pass are presented (C and $\mathbf{D})$. The thrombus in the terminal region of the internal carotid artery was retrieved, but the procedure was continued considering a thrombus to be possibly present in the proximal region of M1.

was 16 , remaining the same as that preoperatively. Cerebral infarction was considered cardiogenic because atrial fibrillation was observed. The patient developed aspiration pneumonia on the eighth hospital day. Tracheal intubation, ventilator management, and antibacterial drug administration were performed, but the patient died on the 13th hospital day.

This case report was approved by the ethics committee of the affiliated institution (approved No. 258).

\section{Discussion}

There are a relatively few normal variants of the MCA. Of these, twig-like MCA (also termed unfused MCA and rete MCA) is relatively rare. ${ }^{1)}$ It has been reported under multiple names. In this study, this pathology was termed twig-like MCA. Many points of the natural history of twiglike MCA remain unclear, and its incidence was reported to be $0.11 \%{ }^{6}$ ) and $1.17 \%{ }^{7)}$ The incidences of other normal variants of the MCA, accessory MCA, duplicated MCA, and fenestrate MCA, have been reported to be $0.3 \%-4.0 \%,{ }^{8,9)} 0.2 \%-2.9 \%,{ }^{8,9)}$ and $0.26 \%,{ }^{8)}$ respectively, demonstrating twig-like MCA to be relatively rare among normal variants of the MCA.

The characteristic imaging findings common to previous reports on twig-like MCA are as follows: 1) steno-occlusive lesion observed in the unilateral MCA, 2) plexiform arterial network is present instead of the MCA trunk, 3) the perforator appears from the plexiform arterial network, and 


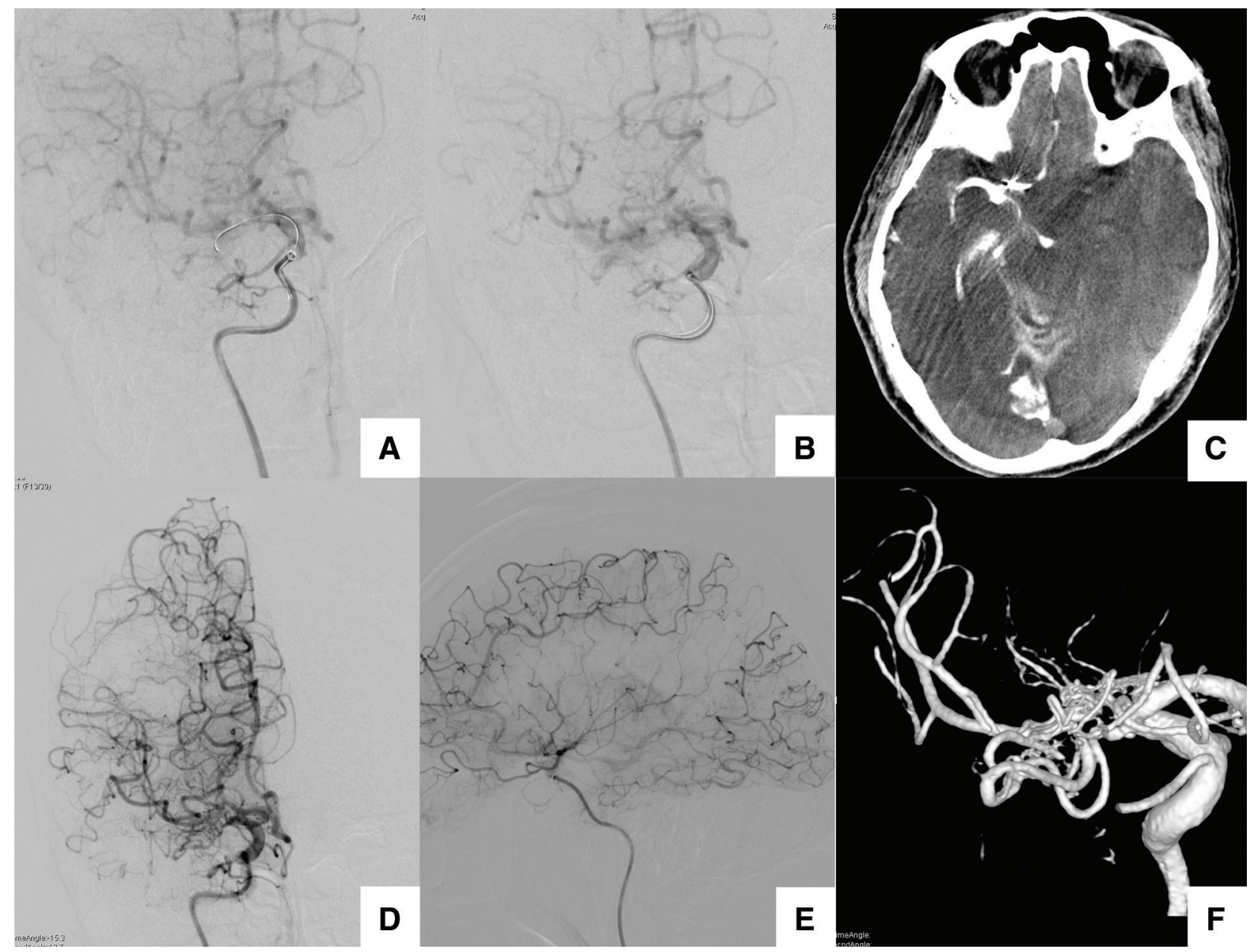

Fig. 3 In the frontal view on angiography, the microguidewire caused perforation when lesion crossing was applied to the thrombus in the proximal region of $\mathrm{M} 1(\mathbf{A})$ and extravasation was observed on contrast imaging (B). In a CT-like image, a small amount of subarachnoid hemorrhage was noted (C). On 3D imaging, the terminal region of the ICA was retained, but it became a plexal blood vessel from the

proximal M1 region of the MCA and the morphology of the distal blood vessel was normal. The frontal and lateral views and threedimensionally reconstructed maximum intensity projection at the final imaging are presented (D-F). It was judged as TICI2b. ICA: internal carotid artery; MCA: middle cerebral artery; $\mathrm{TICl}$ : thrombolysis in cerebral infarction

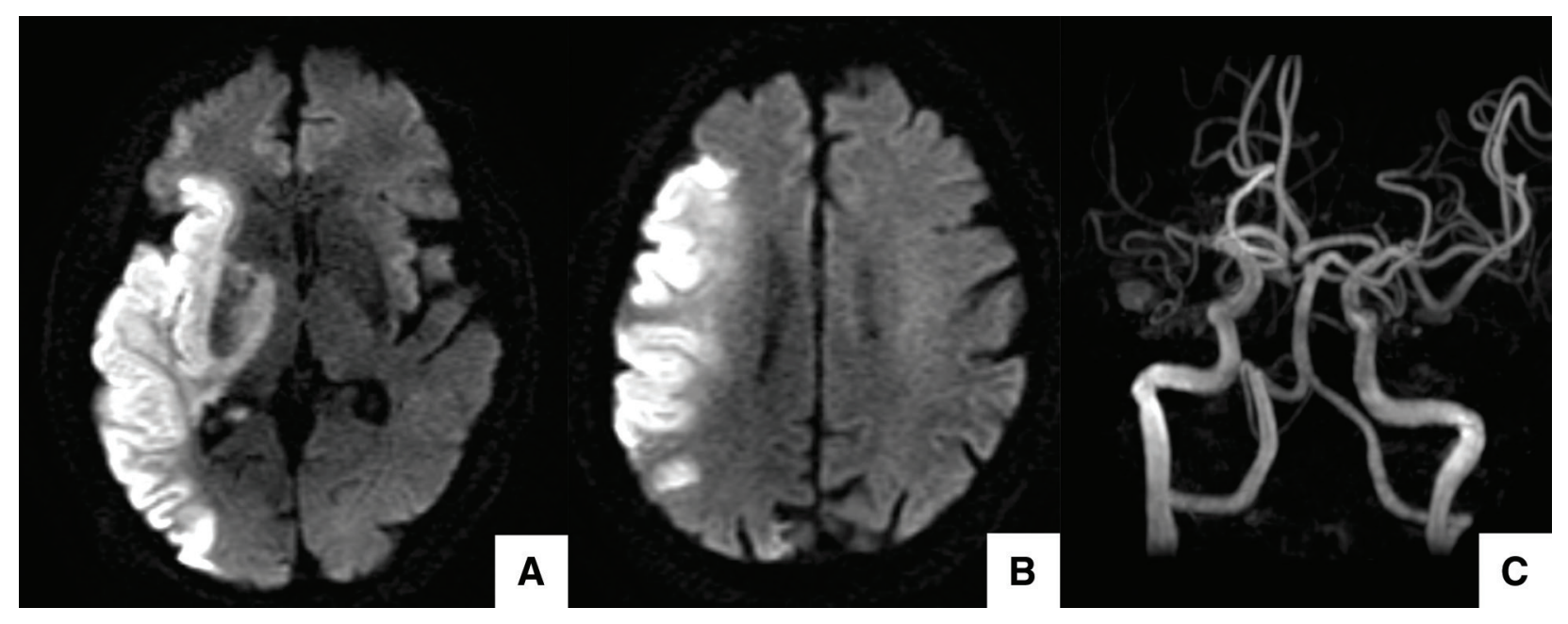

Fig. 4 DWI acquired on the second hospital day. On ASPECTSDWI, the score was 2 (A and $\mathbf{B}$ ). On MRA, terminal occlusion of the right internal carotid artery resolved (C). ASPECTS: Alberta stroke program early CT score; DWI: diffusion weighted imaging 
4) the peripheral cortical branch from the occluded MCA has antegrade blood flow and normal morphology, ${ }^{10)}$ all of which were fulfilled in this case. The pathophysiology of twig-like MCA has not been well clarified. A certain abnormality is considered to result in fusion and regression, thereby forming a twig-like MCA during the process by which the morphology of the primitive plexiform MCA becomes the adult morphology. ${ }^{10)}$ Twig-like MCA causes hemorrhagic stroke and ischemic stroke, but to our knowledge, there has been no report in which twig-like MCA was complicated by ipsilateral acute ICA occlusion and treated by thrombus retrieval. The most important point for percutaneous thrombectomy applied to ipsilateral proximal major cerebral artery occlusion accompanying twig-like MCA is to recognize the pathology, twig-like MCA. The usefulness of a direct aspiration first pass technique $(\mathrm{ADAPT})^{11)}$ for major cerebral artery occlusion was recently reported. ${ }^{12)}$ ADAPT not requiring lesion crossing may be effective in cases with a thin plexal blood vessel distal to the lesion such as this case. In this case, the operators should have recognized the presence of twig-like MCA on angiography (Fig. 2) after the first pass by ADAPT and completed the procedure.

To our knowledge, no case of bilateral twig-like MCA has been reported and it is likely to be difficult to judge twig-like MCA from the morphology of the contralateral MCA in the evaluation of blood vessels on preoperative MRA before thrombus retrieval. However, if the operator evaluates the outer diameter of the blood vessel before surgery, even though the lesion cannot be identified as twiglike MCA, it may be possible to perform percutaneous thrombectomy while being aware that the lesion and blood vessel in the distal region may be thin and lesion crossing with a microguidewire and microcatheter may be difficult. Judgment of the outer diameter of the blood vessel may be possible by evaluating the region around the thrombus region by heavy $\mathrm{T} 2$ weighted image (T2WI) such as 3D-constructive interference in steady state (CISS). ${ }^{13}$ However, there may be fewer facilities that perform evaluation by heavy T2WI in the acute phase of general stroke. In this patient, the outer diameter of the blood vessel was not evaluated by heavy T2WI before surgery and the lesion was unable to be considered twig-like MCA.

Cases complicated by vascular abnormality other than twig-like MCA in a region distal to major cerebral artery occlusion have been reported. In cases of major cerebral artery occlusion, the frequency of occlusion accompanied by unruptured cerebral aneurysm distal to the occluded region discovered during the procedure was $0.8 \%(1 / 124)^{14)}$ to $1 \%(3 / 300)^{15)}$ and that of aneurysm rupture-induced hemorrhagic complication observed during thrombus retrieval was $0.3 \%(1 / 300)^{15)}$ to $0.8 \%(1 / 124) .{ }^{14)}$ To our knowledge, there has been no report of arteriovenous malformation or moyamoya observed at a site distal to the occluded region during the procedure. Although the complication of major cerebral artery occlusion by vascular abnormality is relatively rare, it is necessary to be aware of its presence and pay attention to the vascular anatomy of the region distal to the lesion. In some cases, for example, it may be better to suspend the procedure to prioritize safety even though effective recanalization cannot be acquired.

\section{Conclusion}

Twig-like MCA is a relatively rare entity and its pathology has not been accurately clarified. In this patient, cardiogenic terminal occlusion of the ICA, which occurred in the ipsilateral proximal region of a twig-like MCA, was treated by percutaneous thrombectomy and effective recanalization was achieved, but hemorrhagic complication developed. When percutaneous thrombectomy is performed in cases of twig-like MCA accompanied by major cerebral artery occlusion in the proximal region, it is difficult to guide a microguidewire and microcatheter to the distal region of occlusion, and it is also accompanied by the risk of hemorrhagic complication. For treatment, it is important to be aware of the pathology termed twig-like MCA.

\section{Disclosure Statement}

The authors declare no conflicts of interest.

\section{References}

1) Cho KC, Kim JJ, Jang CK, et al. Rete middle cerebral artery anomalies: a unifying name, case series, and literature review. J Neurosurg 2018; 131: 453-461.

2) Lyden PD, Lu M, Levine SR, et al. A modified National Institutes of Health Stroke Scale for use in stroke clinical trials: preliminary reliability and validity. Stroke 2001; 32: 1310-1317.

3) Hirai T, Sasaki M, Maeda M, et al. Diffusion-weighted imaging in ischemic stroke: effect of display method on observers' diagnostic performance. Acad Radiol 2009; 16 : 305-312.

4) Azizyan A, Sanossian N, Mogensen MA, et al. Fluidattenuated inversion recovery vascular hyperintensities: an 
important imaging marker for cerebrovascular disease. AJNR Am J Neuroradiol 2011; 32: 1771-1775.

5) Zaidat OO, Yoo AJ, Khatri P, et al. Recommendations on angiographic revascularization grading standards for acute ischemic stroke: a consensus statement. Stroke 2013; 44: 2650-2663.

6) Liu HM, Lai DM, Tu YK, et al. Aneurysms in twig-like middle cerebral artery. Cerebrovasc Dis 2005; 20: 1-5.

7) Seo BS, Lee YS, Lee HG, et al. Clinical and radiological features of patients with aplastic or twiglike middle cerebral arteries. Neurosurgery 2012; 70: 1472-1480; discussion 1480.

8) Crompton MR. The pathology of ruptured middle-cerebral aneurysms with special reference to the differences between the sexes. Lancet 1962; 2: 421-425.

9) Komiyama M, Nakajima H, Nishikawa M, et al. Middle cerebral artery variations: duplicated and accessory arteries. AJNR Am J Neuroradiol 1998; 19: 45-49.

10) Uchiyama N. Anomalies of the Middle Cerebral Artery. Neurol Med Chir (Tokyo) 2017; 57: 261-266.
11) Turk AS, Frei D, Fiorella D, et al. ADAPT FAST study: a direct aspiration first pass technique for acute stroke thrombectomy. J Neurointerv Surg 2014; 6: 260-264.

12) Turk AS 3rd, Siddiqui A, Fifi JT, et al. Aspiration thrombectomy versus stent retriever thrombectomy as first-line approach for large vessel occlusion (COMPASS): a multicentre, randomised, open label, blinded outcome, non-inferiority trial. Lancet 2019; 393: 998-1008.

13) Komiyama $M$, Ishiguro $T$, Nishikawa $M$, et al. Constructive interference in steady state imaging of moyamoya disease. Neurol Med Chir (Tokyo) 2002; 42: 11-16; discussion 17.

14) Zhou T, Li T, Zhu L, et al. Endovascular thrombectomy for large-vessel occlusion strokes with preexisting intracranial aneurysms. Cardiovasc Intervent Radiol 2018; 41: 1399-1403.

15) Zibold F, Kleine JF, Zimmer C, et al. Aneurysms in the target vessels of stroke patients subjected to mechanical thrombectomy: prevalence and impact on treatment. $\mathrm{J} \mathrm{Neu}$ rointerven Surg 2016; 8: 1016-1020. 